\title{
An alternative cause of stroke in a young patient with beta-thalassemia
}

\author{
R. Rogans-Watson 1 , S. Mahmood ${ }^{1}$, K. Kee ${ }^{1}$, E. Lawrence 1 . \\ ${ }^{1}$ Croydon University Hospital, Stroke, London, United Kingdom.
}

\section{Croydon Health Services WHS}

NHS Trust

We describe an interesting case of a young man with subacute stroke on a background of beta-thalassemia. The case demonstrates the need to investigate for cardiogenic embolism, instead of assuming hypercoagulability due to iron overload.

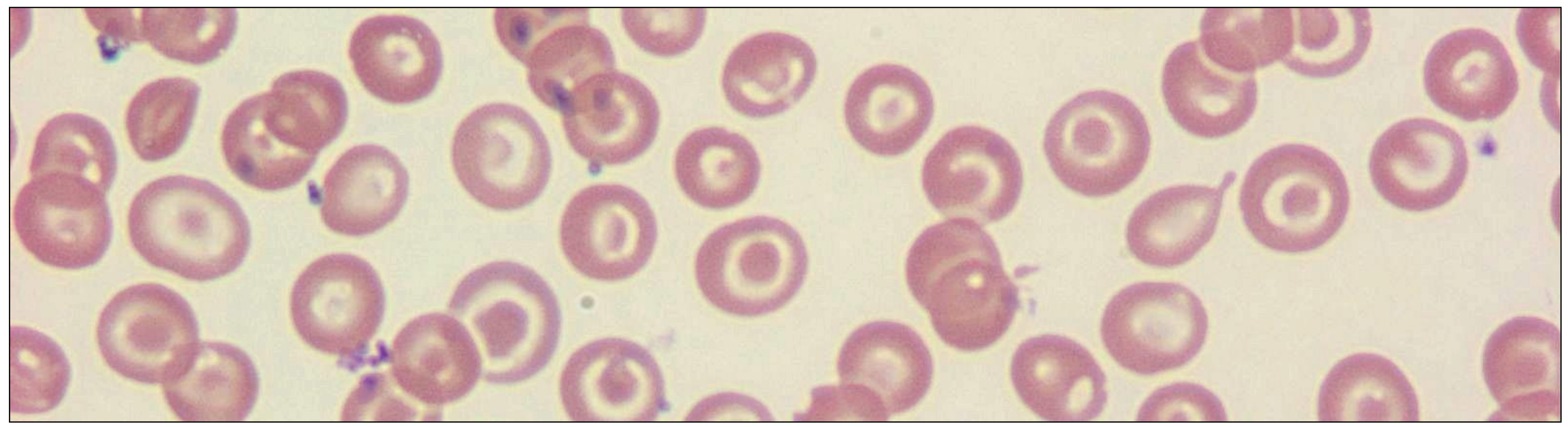

\section{Presentation}

A 35-year-old man was admitted with a 4-day history of left sided weakness and blurred vision in the left eye. He had an associated headache and had vomited once, and had initially attributed the symptoms to a migraine, which led to the late presentation. He had a history of beta-thalassemia, splenectomy, and migraines. He was a non-smoker.

\section{Examination:}

- Reduced visual acuity in the left eye, and mild left sided ptosis

- Mild left sided weakness (MRC 4/5), worse in the lower limb

- Soft systolic murmur, loudest at the apex

\section{Investigations}

- Bloods: macrocytic anaemia (Hb 105g/L, MCV 104.5fL), and raised Ferritin (726 ug/L) and iron saturation (82\%)

- Blood film: target cells, Howell Jolly bodies, and spherocytes (as seen above) in keeping with beta-thalassemia and splenectomy

- Fundoscopy: left inferior branch retinal artery occlusion

- Carotid ultrasound \& MR angiography: normal

- MRI brain: restricted diffusion in anterior limb of the left internal capsule and posterior corpus callosum (see below)

- Echocardiogram: diffuse calcification (thalassemia-induced cardiomyopathy), and a hypermobile mass in the left ventricular outflow tract

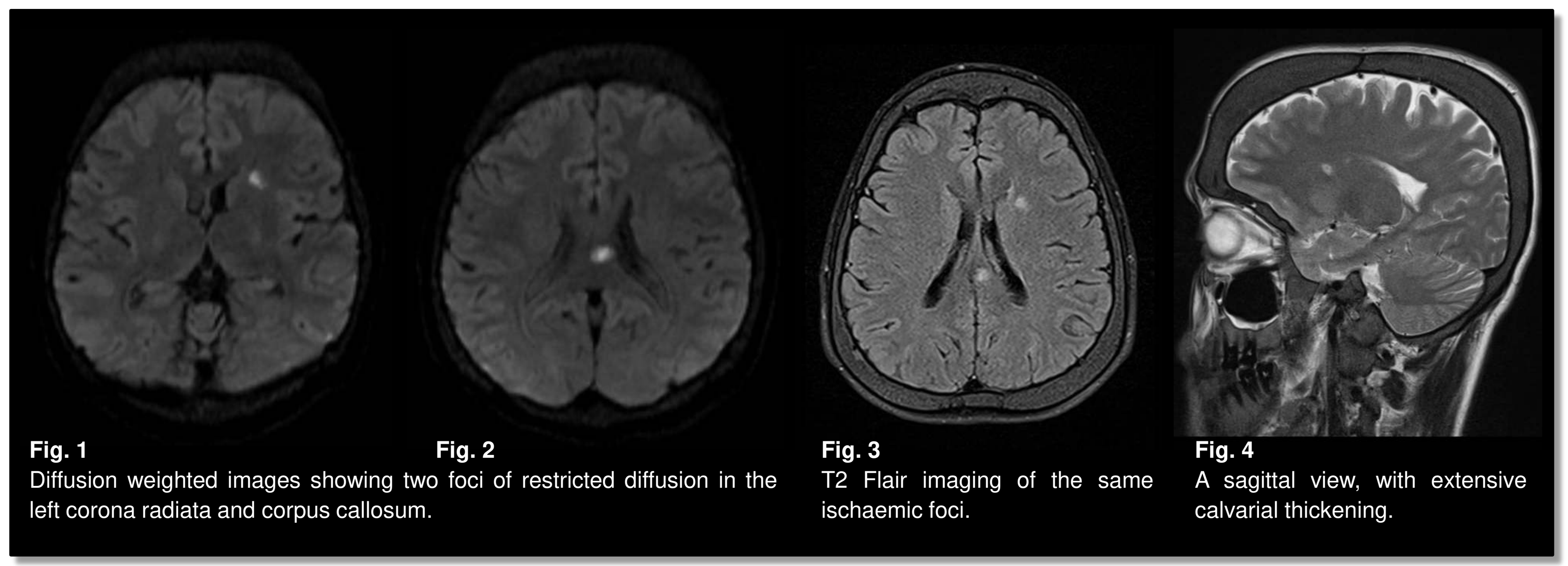

\section{Diagnosis and Management}

The patient was diagnosed with left retinal artery occlusion and a left lacunar stroke, caused by cardiogenic emboli due to thalassemiainduced cardiomyopathy.

His left sided weakness was explained by contralateral pressure on the corpus callosum on the right.

He was commenced on anticoagulation and had no further events.

\section{Conclusion}

Thalassemia does not in itself predispose to stroke, but hypercoagulability-induced thromboembolism due to iron overload is often accepted as causing cerebral ischaemia in thalassemia.

However, this case demonstrates the importance of considering cardiogenic embolism due to siderotoxic cardiomyopathy. Patients with known thalassemia must therefore have urgent cardiac imaging.

Thrombolysis can be applied in the acute setting, and anticoagulation is appropriate for long term management of a ventricular thrombus. 